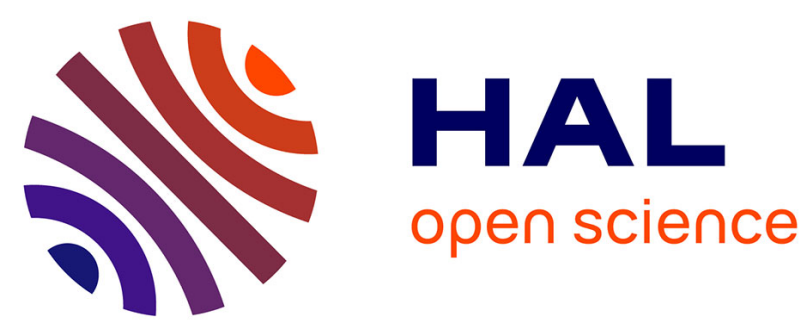

\title{
A Novel Design of Ultrathin Metasurface Structure for Terahertz Time-Domain Spectroscopy (THz-TDS) Applications
}

Mohammad Ojaroudi, Valeria Loscrì

\section{> To cite this version:}

Mohammad Ojaroudi, Valeria Loscrì. A Novel Design of Ultrathin Metasurface Structure for Terahertz Time-Domain Spectroscopy (THz-TDS) Applications. EuCAP 2021 - 15th European Conference on Antennas and Propagation, Mar 2021, Düsseldorf / Virtual, Germany. hal-03082170

\section{HAL Id: hal-03082170 https://hal.science/hal-03082170}

Submitted on 18 Dec 2020

HAL is a multi-disciplinary open access archive for the deposit and dissemination of scientific research documents, whether they are published or not. The documents may come from teaching and research institutions in France or abroad, or from public or private research centers.
L'archive ouverte pluridisciplinaire HAL, est destinée au dépôt et à la diffusion de documents scientifiques de niveau recherche, publiés ou non, émanant des établissements d'enseignement et de recherche français ou étrangers, des laboratoires publics ou privés. 


\title{
A Novel Design of Ultrathin Metasurface Structure for Terahertz Time-Domain Spectroscopy (THz-TDS) Applications
}

\author{
Mohammad Ojaroudi ${ }^{1}$, and Valeria Loscri ${ }^{1}$ \\ ${ }^{1}$ Inria Lille Nord Europe, 40 Av. Halley, 59650 Villeneuve d'ascq, France \\ e-mails: mohammad.ojaroudi-parchin@inria.fr, and valeria.loscri@inria.fr
}

\begin{abstract}
In this paper, an ultrathin metasurface structure is proposed for terahertz time-domain spectroscopy (THz-TDS) applications. The proposed structure consists of an inverted Tshaped strip surrounded by a rectangular-shaped split ring resonator (SRR) structure on a high-resistance silicon substrate. By inserting an inverted T-shaped strip inside the SRR, the coupling between the strip and SRR is improved and the sensitivity (refractive index unit (RIU)) of the proposed unit cell is increased. The proposed unit cell can provide an acceptable transmission characteristic around 0.6 THz. In addition, by using biological analyte, in this study brain Glioma, we are able to detect this material's concentrations by calculating the sensitivity in the range of $65 \mathrm{GHz} / \mathrm{RIU}$. The proposed metasurface structure has small size and high sensitivity characteristics. Simulation results of the transmission coefficients and radiation characteristics show the effectiveness of the proposed design for biomedical applications.
\end{abstract}

Index Terms - terahertz time-domain spectroscopy (THzTDS), attenuated total reflectance (ATR) spectroscopy, ultrathin metasurface structure, split ring resonator $(S R R)$, inverted T-shaped strip.

\section{INTRODUCTION}

In recent years there have been a lot of researches on the using of terahertz time-domain spectroscopy (THz-TDS) method in the early diagnosis of brain diseases such as cancerous tumors, traumatic injuries, Parkinson's and Alzheimer's diseases [1-2]. In these applications one of the most notable use of THz-TDS is separating healthy brain tissue from damaged tissues [3-4]. For example, THz-TDS is used for detecting brain tumors by measuring the amount of Glioma matter in brain tissue [5].

Metasurface structure as a 2D structure of metamaterials has been used extensively in the range of terahertz imaging and communication applications [6-7]. Metasurface has found a variety of applications in areas such as holographic imaging, TDS, biosensors [8-9]. Due to the fact that high-sensitivity structures can be created using metasurface, the improvement of TDS by using metasurface layers has been widely used in the initial diagnosis of cancer [10]. In addition, regarding to their potential characteristics, such as their flexible structure, small dimensions can be realized with a variety of materials such as silicon and graphene, with the ability to simultaneously control the size and phase of the return waves.

This paper presents a new ultrathin metasurface structure based on an inverted T-shaped strip surrounded by SRR for using in biosensing applications in THz-TDS. By inserting the inverted T-shaped strip inside the SRR structure, we can improve the coupling between the inverted T-shaped strip and the SRR, with an improved transmission impact at resonance frequency of $0.6 \mathrm{THz}$. To show this property, the transmission coefficient and surface current distributions are given at this frequency. In addition, a parameter sweep study has also been performed to show how the frequency can be shifted by changing the gap distance between the inverted Tshaped strip and SRR. The proposed structure is simple and easy to build since it is designed on a silicon substrate with the thickness of $50 \mu \mathrm{m}$ and aluminum metal with $20 \mu \mathrm{m}$. The simulation results show that the proposed structure has 65 $\mathrm{GHz} / \mathrm{RIU}$ sensitivity factor which proves that the proposed metasurface structure is a good candidate for biosensing applications in THz-TDS.

The rest of the paper is organized as follows. In Section II we present the metasurface design for THz-TDS applications. In Section III we detail the parametric sweep analysis and surface current distributions of the proposed structure. Finally, we conclude the paper in Section IV.

\section{Design AND CONFIGURATION OF THE PROPOSED METASURFACE FOR THZ-TDS APPLICATIONS}

The schematic of THz-TDS and ATR-TDS setups are shown in Fig. 1. As shown in Fig. 1, the optical beam is converted to terahertz beam by a converter such as nano-antennas. After passing the metasurface/analyte, the passing wave is recorded by the $\mathrm{THz}$ detector. In the simulation, the scattered $\mathrm{THz}$ wave is radiated to the top layer of the metasurface structure which is covered by an analyte. Presence of this analyte will change the transmission which leads to understand the amount/concentration of analyte by changing the received signal's spectrum [11]. In this paper we focused on attenuated total reflectance (ATR) spectroscopy [12]. In ATR based THz-TDS a high resistance silicon prism is used to gather the reflected wave, as shown in Fig. 1(b).

The proposed unit cell structure is shown in Fig 2 (a). In this structure we proposed an inverted T-shaped microstrip structure enclosed by SRR. In the proposed structure, the aluminum metal with the thickness of $20 \mu \mathrm{m}$ is printed on a silicon substrate with thickness of $50 \mu \mathrm{m}$. The electrical conductivity of aluminum is $\sigma=3.56 \times 10^{7} \mathrm{~S} / \mathrm{m}$, the dielectric constant of the high resistance silicon substrate with the thickness of $50 \mu \mathrm{m}$ is $\varepsilon_{r}=11.9$, and its resistivity $\gamma=6000 \Omega$. 
Cm [10]. All dimensions of the proposed unit-cell configuration are summarized in Table I.

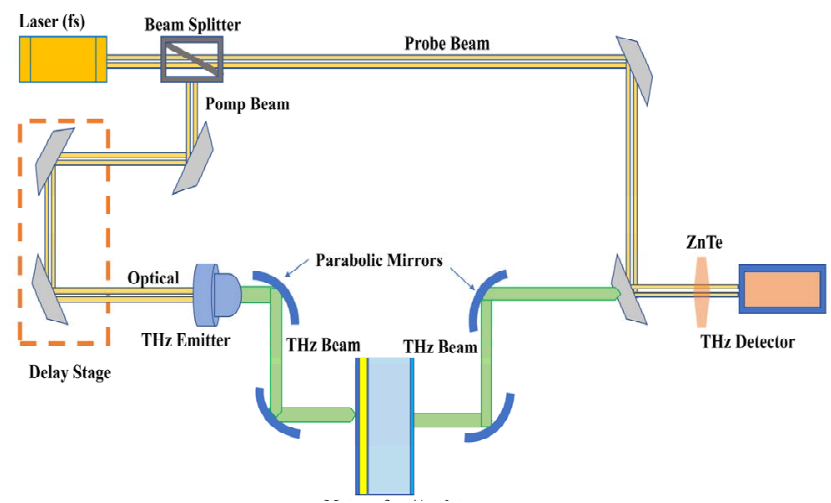

(a)

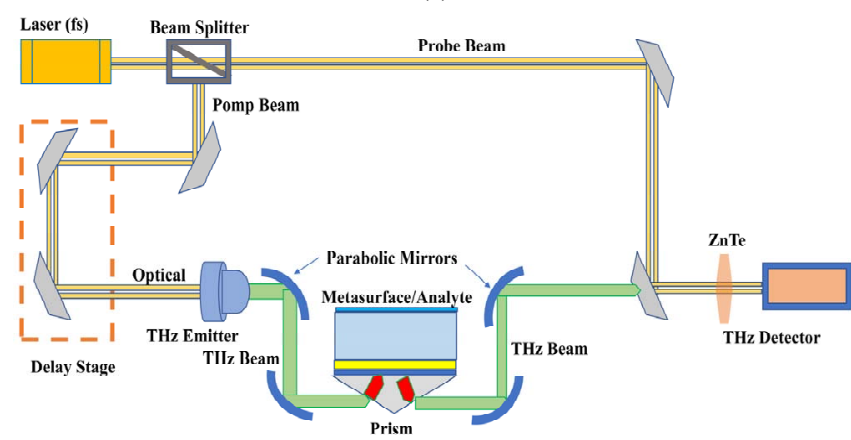

(b)

Fig. 1. THz time-domain spectroscopy setups for highly-sensitive applications (a) THz-TDS method, and (b) Attenuated total reflectance (ATR) spectroscopy.

In addition, the extracted equivalent circuit for the proposed metasurface structure is shown in Fig. 2 (b). In this structure, the air gaps are modelled as capacitors. In addition, to model the effects of surface current on aluminum metal the inductors are used. As demonstrated in equivalent circuits, the combination of these capacitors with the indicators has created four LC series resonators. By changing the air gap that changes the capacitance of the capacitor in the resonator, significant changes in the frequency response of the overall structure are obtained [13].

Side View

Top View
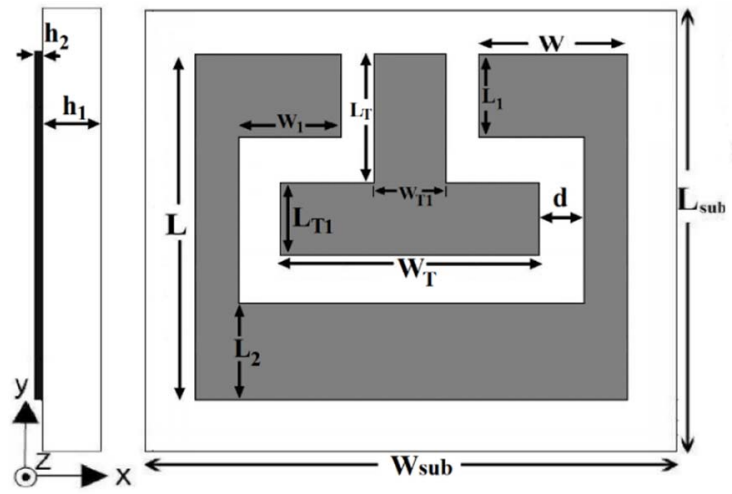

(a)

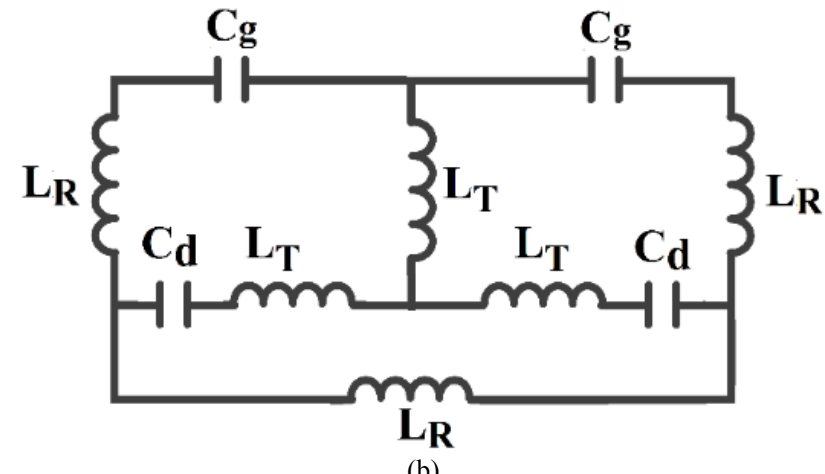

(b)

Fig. 2. The proposed unit cell with a split ring resonator (SRR) with inverted T-shaped strip, (a) The configuration and the geometry, and (b) the extracted conceptual equivalent circuit model.

TABLE I THE PROPOSED UNIT CELL DIMENSIONS

\begin{tabular}{|c|c|c|c|c|c|}
\hline Parameter & $\mu \mathrm{m}$ & Parameter & $\mu \mathrm{m}$ & Parameter & $\mu \mathrm{m}$ \\
\hline $\mathrm{W}_{\text {sub }}$ & 60 & $\mathrm{~L}_{\text {sub }}$ & 60 & $\mathrm{~L}$ & 50 \\
\hline $\mathrm{L}_{2}$ & 15 & $\mathrm{~W}$ & 15 & $\mathrm{~W}_{\mathrm{T}}$ & 22 \\
\hline $\mathrm{L}_{1}$ & 15 & $\mathrm{~W}_{1}$ & 10 & $\mathrm{~d}$ & 11 \\
\hline $\mathrm{L}_{\mathrm{T}}$ & 20 & $\mathrm{~L}_{\mathrm{T} 1}$ & 10 & $\mathrm{~W}_{\mathrm{T} 1}$ & 10 \\
\hline $\mathrm{h}_{1}$ & 20 & $\mathrm{~h}_{2}$ & 200 & & \\
\hline
\end{tabular}

\section{RESULTS AND DISCUSSION}

In this section a parametric sweep analysis and surface current distributions of the proposed structure is demonstrated to show the effective usefulness of this structure for THz-TDS applications.

\section{A. Metasurface Unit Cell Parametric Sweep Analysis}

The proposed unit cell is simulated by CST simulator [14] where unit cell for the boundary condition in $\mathrm{x}$ - and $\mathrm{y}$ directions and open-add space in $z$ direction are selected. The "adaptive mesh refinement" method is selected for meshing. Fig. 3 compares the transmission characteristics of the proposed structure with and without inverted T-shaped strip. As depicted in Fig. 3, there is an operation narrow-band at 0.6 $\mathrm{THz}$ and at the out of desired band, the amplitude is significantly affected by the modified inverted T-shaped parasitic structure.

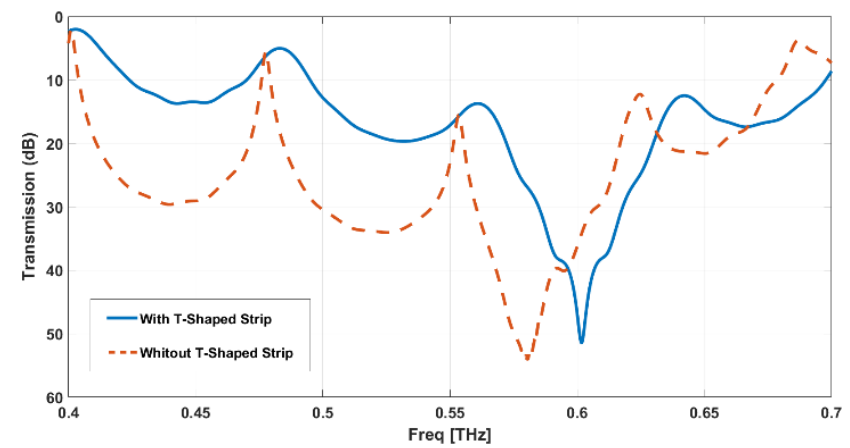

Fig. 3. Comparison of simulated transmission results for the proposed unit cell with and without T-shaped strip.

In order to understand the phenomenon behind using the invreted T-shaped strip effects on performance enhancment of the proposed unit cell, the simulated current distributions 
on the metasurface radiating patch at $0.6 \mathrm{THz}$ (the central resonance frequency) whithout using analyte are plotted in Fig. 4. Due to the resonant properties of the proposed unit cell, the transmission characteristics change at this frequency. Therefore, as shown in Fig. 4, the current is concentrated around the SRR and T-shaped strip's corners at resonance frequency [15]. In addition, to evaluate the effect of gap distance in the performance of the proposed unit cell Fig. 5 shows the simulated transmission results for the proposed unit cell for different values of gap distance d. As shown in Fig. 5, the central frequency and the minimum value of transmission can be controlled by changing the gap distance.

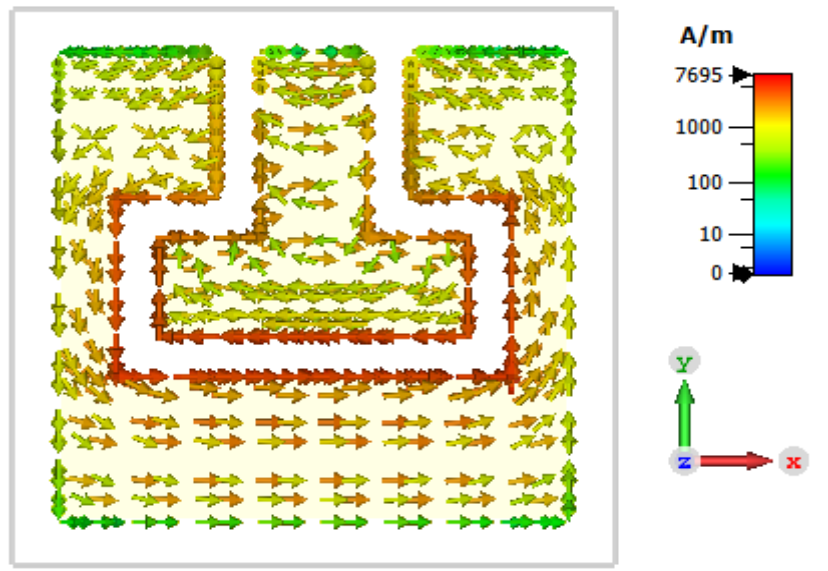

Fig. 4. Simulated surface current distribution for the proposed metasurface unit cell structure at $0.60 \mathrm{THz}$.

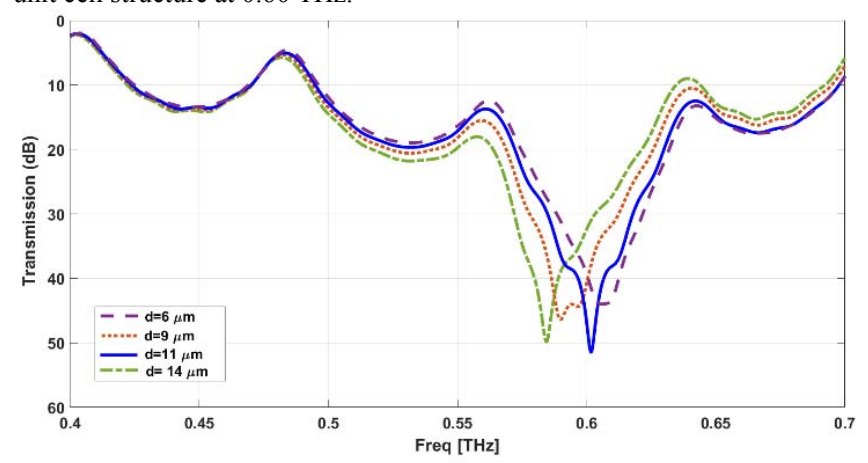

Fig. 5. Simulated transmission results for the proposed unit cell for different values of gap distance (d).

\section{B. High-Sensitivity Analysis using Brain Glioma Analyte for Traumatic Brain Injury Applications}

In this section, in order to perform high sensitivity analysis the surface of the metasurface structure is covered by an appropriate analyte. In this context we added a layer of brain glioma analyte with the thickness of t. Fig. 6 shows the proposed unit cell including analyte simulated in floquet mode and associated boundary condition.

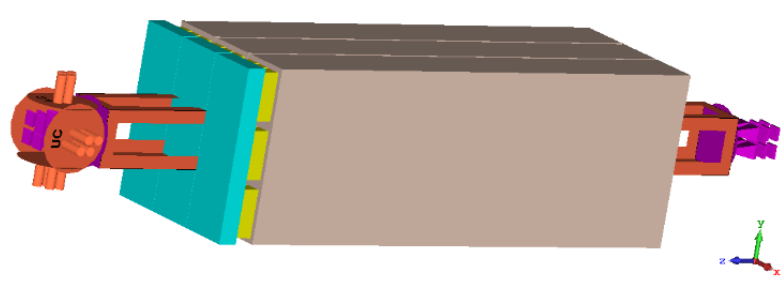

Fig. 6. The proposed unit cell including analyte simulatd in floquet mode and associated boundary condition.

To demonstarte the effect of the proposed analyte thickness on transmission results, Fig. 7 shows the simulated transmission results for the proposed unit cell for different values of analyte thickness t. As demonstrated in Fig. 7 by inctreasing the thickness of analyte layer the frquency is shiftted to higher frequncies. The selected thickness for the alanyte is $10 \mu \mathrm{m}$ to cover the frequency range of $0.6-0.7 \mathrm{THz}$.

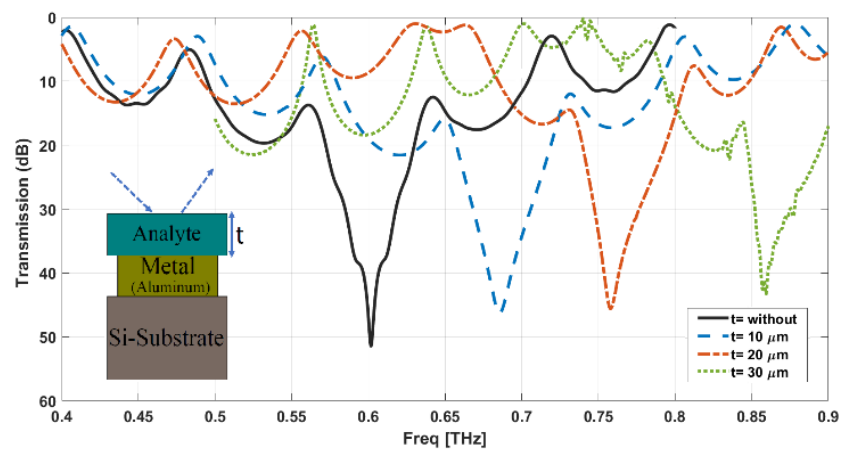

Fig. 7. Simulated transmission results for the proposed unit cell for different values of analyte thickness $t$.

Another parameter that has been studied here is the sensitivity factor. This factor is an important parameter in the biosensing applications and for most of materials is less than $60 \mathrm{GHz} / \mathrm{RIU}$ [16]. This parameter is extracted from frequency shift due to the shifting on refractive index. The refraction index $\mathrm{n}$ can be calculated from permittivity $\varepsilon$ and permeability $\mu$ of materials as follow:

$$
\varepsilon=n^{2} / \mu
$$

For the materials which are used in this paper such as silicon, aluminum and brain glioma, the permeability $\mu=1$. It can be understood from equation (1) by increasing the permittivity, the refraction index will be increased. Based on this truth in this study in order to model the brain glioma analyte with different refraction index we used different permittivity for the analyte. In this point of view the sensitivity factor can be calculated as follow:

$$
S=\delta f / \delta n
$$

where, $\delta \mathrm{f}$ is the resonance frequency shifting, and $\delta \mathrm{n}$ is the difference of refractive index based on changing primitivity of the selected analyte, respectively [10].

Fig. 8 shows the transmission characteristics versus changing refractive index. From Fig. 8 the $\delta$ f and $\delta$ n can be extracted. Hence, the sensitivity factor $\mathrm{S}$ can be determined. In this Figure the metasurface is coated with a layer of 20$\mu \mathrm{m}$-thick Glioma analytes with different values for refractive 
index, i.e., $n=1,1.5,1.75$, and 2. As illustrated in Fig. 8 the proposed metasurface has the capability to reaches up to 65 $\mathrm{GHz} / \mathrm{RIU}$ which means, the designed structure is highly sensitive to the alteration of its surface which is covered by the analyte.

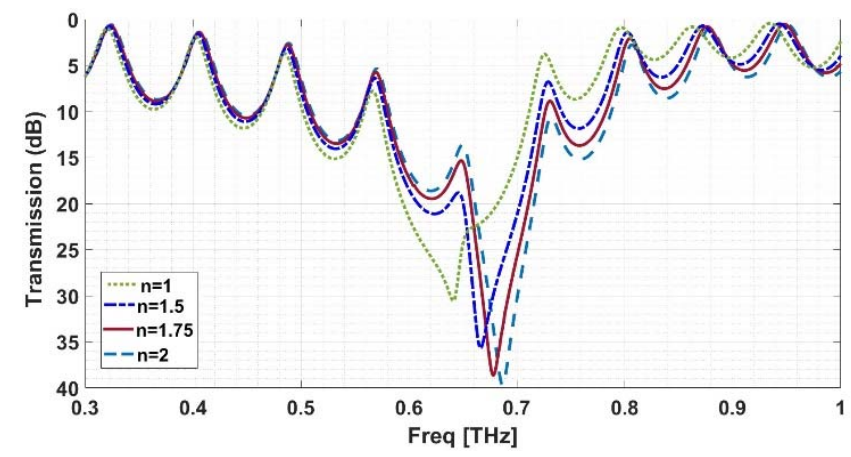

Fig. 8. Simulated transmission results for the proposed unit cell for different values of analyte refractive index $n$.

For study on array structure of the proposedunit cell, Fig. 9 shows two different array configurations of the proposed metasurface structure to demonstrate of the possibility of these structure to cover brain glioma samples. We arrange four unit-cells in $2 \mathrm{D}$ square configuration $(2 \times 2$ metasurface array) which is simulated using CST simulator to demonstrate the radiation characteristics. In this simulation, the metasurface is located in the X-Y plane, and two wave ports are used as an THz-EM sources, which are located at $(0 \mu \mathrm{m}, 0 \mu \mathrm{m}, \pm 20 \mu \mathrm{m})$.
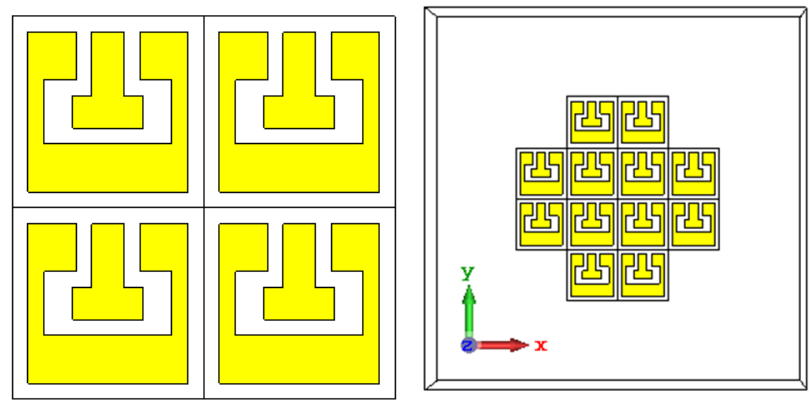

Fig. 9. Two different array configurations of the proposed metasurface structure to cover brain glioma samples.

Fig. 10 shows the simulated time domian signlas including incident, transmitted and reflected signals which shows the effect of attenuation also the delay related to the metasurface with analyte. Strong reflection confirms poor transmission. As shown in the figure, after a few picoseconds, most of the incident wave is reflected.

In addition, Fig. 11 shows the simulation results of $2 \mathrm{D}$ and $3 \mathrm{D}$ radiation patterns including realized gain for $2 \times 2$ array structure, (a) port 1, and (b) port 2. As shwon in Fig. 11 the radiation patterns are omindirectional from both waveports uniformly. In addition, Fig. 12 shows the simulation results of focusing beam in metasurface, which confirm the uniform distribution of the wave on the analyte surface.

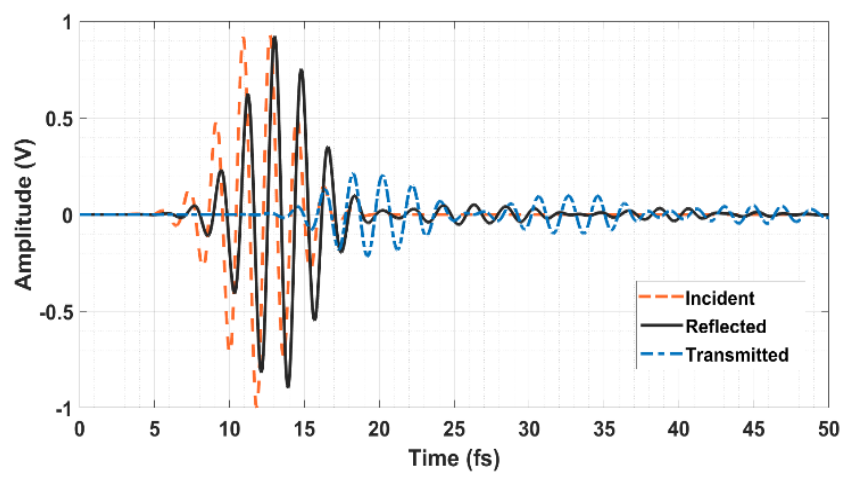

Fig. 10. Time domian incident, transmitted and reflected signals.

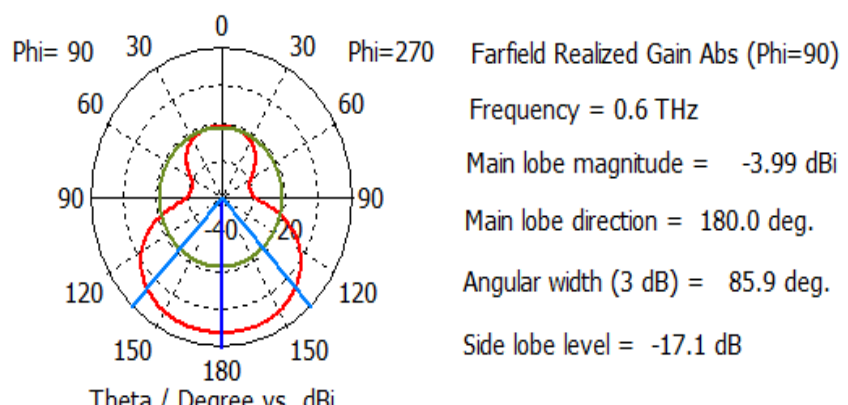

(a)
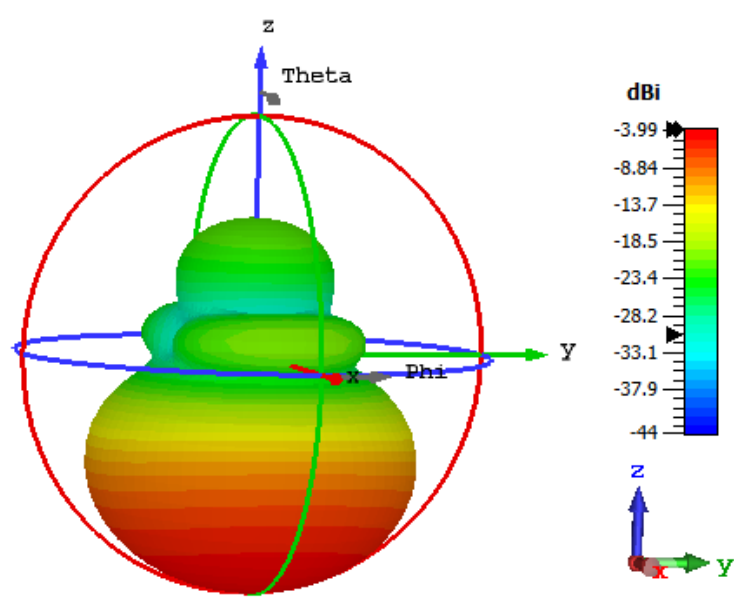

(b)

Fig. 11. The simulation results of $2 \mathrm{D}$ and $3 \mathrm{D}$ radiation patterns including realized gain for $2 \times 2$ array structure, (a) port 1 , and (b) port 2 .

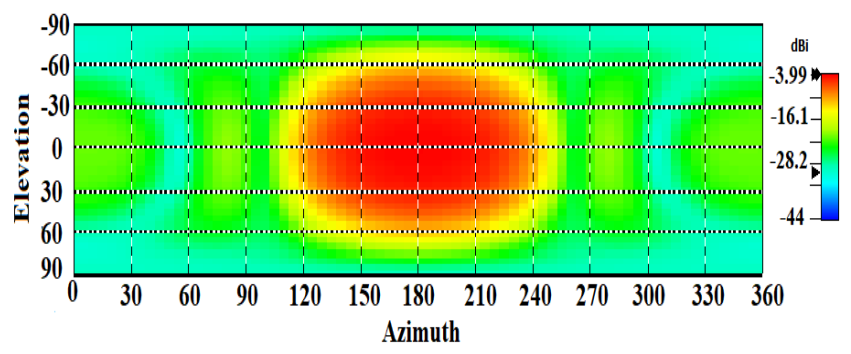

Fig. 12. The simulation results of focusing beam in metasurface. 


\section{CONCLUSION}

In this paper, a new design of highly sensitive metasurface based biosensor structure is presented for terahertz ATR-TDS applications. The structure is covered on a silicone layer with a thin layer of aluminum with a thickness of 20 micrometers. By inserting the inverted T-shaped strip we could improve the coupling between the inverted T-shaped strip and the SRR, which has improved the transmission results at resonance frequency of $0.6 \mathrm{THz}$. First, parameters related to the single structure such as frequency response and surface flow distribution are presented. Then, using an analysis such as brain tissue, parameters related to changes in the thickness of the analyte layer and its refractive index are examined. The simulated results provide a good feature of the proposed structure for biosensing applications.

\section{REFERENCES}

[1] L. Yu, L. Hao, T. Meiqiong, H. Jiaoqi, L. Wei, D. Jinying, C. Xueping, F. Weiling, and Z. Yang, "The medical application of terahertz technology in non-invasive detection of cells and tissues: opportunities and challenges," RSC advances, 9(17), pp.9354-9363, 2019.

[2] W. G. Yeo, O. Gurel, N. Srinivasan, P. D. King, N. K. Nahar, S. Park, N. L. Lehman, and K. Sertel, "Terahertz imaging and electromagnetic model of axon demyelination in Alzheimer's disease," IEEE Transactions on Terahertz Science and Technology, 7(6), pp.711-721, 2017.

[3] P. U. Jepsen, D. G. Cooke, and M. Koch, "Terahertz spectroscopy and imaging-Modern techniques and applications," Laser \& Photonics Reviews, 5(1), pp.124-166, 2011.

[4] P. Yan, W. Chen, and Y. Zhu. "Identification of Biomarker (L-2HG) in Real Human Brain Glioma by Terahertz Spectroscopy," In 2018 Conference on Lasers and Electro-Optics (CLEO), pp. 1-2. IEEE, 2018.

[5] K. Meng, T.N. Chen, T. Chen, L.G. Zhu, Q. Liu, Z. Li, F. Li, S. C. Zhong, Z. R. Li, H. Feng, and J. H. Zhao, "Terahertz pulsed spectroscopy of paraffin-embedded brain glioma," Journal of biomedical optics, 19(7), p.077001, 2014.

[6] A. E. Yachmenev, D.V. Lavrukhin, I.A. Glinskiy, N. V. Zenchenko, Y.G. Goncharov, I. E. Spektor, R. A. Khabibullin, T. Otsuji, and D. S. Ponomarev, Metallic and dielectric metasurfaces in photoconductive terahertz devices: a review. Optical Engineering, 59(6), p.061608. 2019.

[7] S. Sun, Q. He, J. Hao, S. Xiao, and L. Zhou, "Electromagnetic metasurfaces: physics and applications," Advances in Optics and Photonics, 11(2), pp.380-479. 2019.

[8] H. C. Liu, B. Yang, Q. Guo, J. Shi, C. Guan, G. Zheng, H. Mühlenbernd, G. Li, T. Zentgraf, and S. Zhang, "Single-pixel computational ghost imaging with helicity-dependent metasurface hologram," Science advances, 3(9), p.e1701477, 2017.

[9] Y. Zou, J. Li, Y. Cui, P. Tang, L. Du, T. Chen, K. Meng, Q. Liu, H. Feng, J. Zhao and M. Chen, "Terahertz spectroscopic diagnosis of myelin deficit brain in mice and rhesus monkey with chemometric techniques," Scientific Reports, 7(1), pp.1-9, 2017.

[10] D. Li, S. Lin, F. Hu, Z. Chen, W. Zhang, and J. Han, "Metamaterial Terahertz Sensor for Measuring Thermal-Induced Denaturation Temperature of Insulin," IEEE Sensors Journal, 20(4), pp.1821-1828, 2019.

[11] R. Peretti, S. Mitryukovskiy, K. Froberger, M.A. Mebarki, S. Eliet, M. Vanwolleghem, and J. F. Lampin, "THz-TDS time-trace analysis for the extraction of material and metamaterial parameters," IEEE transactions on Terahertz Science and Technology, 9(2), pp.136-149. 2018.

[12] Y. Zhong, L. Du, Q. Liu et al., "Metasurface-enhanced ATR sensor for aqueous solution in the terahertz range", Optics Communications, 2020 .
[13] Zhang, Z., Ding, H., Yan, X., Liang, L., Wei, D., Wang, M., Yang, Q. and Yao, J., 2018. Sensitive detection of cancer cell apoptosis based on the non-bianisotropic metamaterials biosensors in terahertz frequency. Optical Materials Express, 8(3), pp.659-667.

[14] CST Microwave Studio. ver. 2019, CST, Framingham, MA, USA, 2019.

[15] Singh, R., Al-Naib, I.A., Koch, M. and Zhang, W., 2010. Asymmetric planar terahertz metamaterials. Optics express, 18(12), pp.1304413050 .

[16] Liu, W., Fan, F., Chang, S., Hou, J., Chen, M., Wang, X. and Bai, J., 2017. Nanoparticles doped film sensing based on terahertz metamaterials. Optics Communications, 405, pp.17-21. 